\title{
Interspecific Plasmid Transfer and Modification of Heat-Stable Enterotoxin Expression by Klebsiella pneumoniae from Infants with Diarrhea
}

\author{
MARIA ALESSIO, FABIO ALBANO. LUIGI TARALLO, AND ALFREDO GUARINO
}

Department of Pediatrics. University of Naples, Italy

\begin{abstract}
We have previously described two heatstable enterotoxins (ST) produced by Citrobacter freundii and by Klebsiella pneumoniae. To see whether transfer of toxigenic ability may occur between different bacterial species, conjugal mating experiments were performed using strains isolated from children. Donors and recipients were incubated together. ST production by the recipients was tested by the suckling mouse assay, which is the standard biologic method for detecting ST, and also by an ELISA test. The latter is based on MAb directed to Escherichia coli 18- or 19-amino acid toxin. Citrobacter and $E$. coli strains producing an ELISA-positive ST were used as plasmid donors. Recipients were E. coli, Klebsiella P89, and Klebsiella AL55. The latter had been previously shown to be capable of producing an ELISA-negative ST, but had spontaneously lost this ability. After conjugation, all strains gave positive results in the suckling mouse assay. However, E. coli and Klebsiella P89 ST were positive in the ELISA test, whereas Klebsiella AL55 ST was negative. The same three strains were transformed by inserting the plasmid pSLM004 encoding an ELISA-positive ST. Klebsiella $\mathrm{P89}$ and $E$. coli became capable of producing an ELISA-positive ST, whereas Klebsiella AL55 cells were positive in the suckling mouse assay but negative in the ELISA. To assess whether the toxigenicity acquired was itself transmissible, the new ST-producing strains were incubated with a nontoxigenic $C$ itrobacter strain. The latter became capable of producing an ELISA-positive ST in all cases. In conclusion: $I$ ) different enterobacteria may transmit ST plasmids by conjugation; 2) the ability to produce $\mathrm{ST}$ is acquired together with the ability to transfer it to other bacteria; and 3 ) selected strains of Klebsiella are capable of modifying toxin expression through a posttranslational, yet unknown, mechanism. (Pediatr Res 33: 205208, 1993)
\end{abstract}

\section{Abbreviations}

ST, heat-stable enterotoxin

SMA, suckling mouse assay

Escherichia coli that produce heat-stable toxins are among the most common causes of infantile diarrhea both in developing (1) and in industrialized (2) countries.

The E. coli ST1 or STa comprises a family of low-molecularweight peptides capable of inducing diarrhea. The genes for ST1

Received May 19. 1992: accepted September 24, 1992.

Correspondence and reprint requests: Alfredo Guarino, M.D., via S. Pansini 5. 80131 Naples, Italy.

Supported by a grant from Consiglio Nazionale delle Ricerche Target Project "Biotechnology and Bioinstrumentation, research program 91.01231.P.F.70. are usually located in plasmids (3). Two prototypes of ST1 exist, with minor differences in gene and amino acid sequences (4). The first is an 18-amino acid peptide and is named STIa (or STp). The second, STlb (or STh), is a 19-amino acid peptide. However, ST of larger molecular size have also been described. and they probably represent pretoxin forms (5). Both ST la and ST $1 \mathrm{~b}$ induce diarrhea in man through a multistep process involving the binding of the toxin to receptors located on enterocytes $(6)$ and the coupled stimulation of guanylate cyclase activity (7). ST-like enterotoxins may be produced by bacteria other than E. coli, including nonagglutinating Vibrio (8) and Yersinia enterocolitica (9). These toxins share with $E$. coli ST1 a segment of 13 amino acids, which contains six cysteine residues and is responsible for the biologic activity (10). We have previously described two ST-like enterotoxins produced by Citrobacter freundii (11) and by Klebsiella pneumoniae (12). The first is identical to the 18-amino acid $E$. coli ST1a (13). Klebsiella toxin is less characterized: its physicochemical features are similar to those of $E$. coli ST la; like $E$. coli ST 1a, Klebsiella ST stimulates guanylate cyclase activity and induces net intestinal secretion of water and chloride (12). However, its time-course effect is shorter than that of $E$. coli ST. Furthermore, Klebsiella ST does not react with $\mathrm{MAb}$ raised against pure $E$. coli 18 -amino acid ST1a in an ELISA assay (12). Both toxigenic Citrobacter and Klebsiella strains were isolated from infants with acute watery diarrhea (11, 12). A toxigenic Citrobacter strain was isolated from a patient also harboring an ST-producing E. coli strain (11). This raises the possibility of in vivo transfer of toxigenic ability among different bacterial species.

To find evidence of an interspecific transfer of virulence properties, bacterial conjugal mating as well as transformation experiments were carried out using wild strains isolated from children.

\section{MATERIALS AND METHODS}

The following strains were used as ST plasmid donors in bacterial mating experiments: 1) $C$. freundii AG2ST capable of producing an 18-amino acid ST, isolated from a child with diarrhea (11); 2) E. coli R18 (producing an 18-amino acid ST), isolated from a child with diarrhea; and 3) E. coli R19 (producing a 19-amino acid ST) (14).

Recipients strains were: I) E. coli $\mathrm{K} 12 \mathrm{Rif}^{\mathrm{r}} \mathrm{Lac}-$ (resistant to rifampicin and nonlactose fermenting); 2) $K$. pneumoniae P89 isolated from the stools of a healthy child; 3) $\mathrm{K}$. pneumoniae AL55, isolated from a child with secretory diarrhea (12), and 4) C. freundii strain FA29, isolated from a healthy child. K. pneumoniae AL55 had been previously shown to produce an ELISAnegative ST, but subsequently had spontaneously lost this ability. Citrobacter strain was also used as recipient in the remating experiments.

Experimental procedure. Donor and recipient cultures, grown in $\mathrm{L}$ broth $(10 \mathrm{~g}$ Tryptone, $5 \mathrm{~g}$ yeast extract, and $5 \mathrm{~g} \mathrm{NaCl} / \mathrm{L}, \mathrm{pH}$ 7.2) at $37^{\circ} \mathrm{C}$, in shaking incubation, were mixed in a volume 
ratio of $1: 2$ and incubated for $2 \mathrm{~h}$ at $37^{\circ} \mathrm{C}$. Mixed culture was plated onto xylose lysine desoxycholate agar. This was supplemented with $100 \mu \mathrm{g} / \mathrm{mL}$ of rifampicin when $E$. coli K12 Rif ${ }^{r}$ $\mathrm{Lac}$ - was used as the recipient. The other recipients were selected on the basis of morphology and lactose fermentation and/or $\mathrm{H}_{2} \mathrm{~S}$ production. Identification was confirmed by a multitest system (API $20 \mathrm{E}$ ). At least 20 pools, each containing five different colonies, were obtained from each mating and tested for enterotoxigenicity. ST production was tested by the SMA (15): the SMA was performed by killing the animals 150 min after jejunal inoculation of culture supernatant (12). ST-positive and STnegative control strains were used in each experiment. Results were considered positive when the gut weight/body weight ratio exceeded $0.083(15)$. ST production was also tested by an ELISA test, which uses MAb raised against pure 18-amino acid $E$. coli ST (16). This ELISA test is capable of detecting both 18- and 19 amino acid ST.

Transfer frequencies were expressed as the ratio of the number of transformed (ST-positive) cells to the number of recipient cells. This was done by testing serial 5-fold dilutions of recipient cells for the production of ST by the SMA.

Transformation experiments were performed as described (17), by incubating competent Klebsiella cells with the plasmid pSLMOO4 from $E$. coli $\mathrm{HB} 101$, carrying a gene encoding for 19-amino acid ST1 and a gene for ampicillin resistance (14). Transformed cells were selected by their growth on LB agar supplemented with $200 \mu \mathrm{g} / \mathrm{mL}$ of ampicillin, to which Klebsiella AL55 was originally sensitive, and successively tested for ST production. Physicochemical characteristics of ST toxins, including heat stability, $\mathrm{pH}$ inactivation, methanol solubility, and mercaptoethanol sensitivity, were determined as previously described (12). Molecular weight was estimated by testing filtrate and retentate fractions of ultrafiltration membranes (PM-10 and $\mathrm{UM}-2$ ) in the SMA (12).

Stability of enterotoxin production was tested each month on culture supernatant prepared from strains kept in nutrient agar at room temperature in the dark.

Culture media were purchased from Difco s.a.s., Milan, Italy. API system $20 \mathrm{E}$ was obtained from Ayerst Italiana, Milan, Italy. Ultrafiltration membranes were purchased from Amicon Corp., Lexington, MA. Chemicals were obtained from Sigma Chemical Co., St. Louis, MO. Anti-ST MAb were a gift from Dr. R. A. Giannella, Division of Digestive Diseases, University of Cincinnati, OH. E. coli R 18 was obtained from Dr. A. Caprioli, I.S.S. Rome, Italy. E. coli HB 101 containing the plasmid pSLM004 was obtained from Dr. A. Fasano, University of Reggio Calabria, Italy. This strain was renamed R19. Serotyping of Klebsiella strains was kindly performed by Fritz and Ida Orskov, International Escherichia and Klebsiella Centre, Statens Seruminstitute, Copenhagen. Denmark.

\section{RESULTS}

Conjugal transfer of ST production. Results of bacterial conjugal matings are reported in Table 1 . All matings resulted in the transfer of toxigenic ability to recipients. However, $E$. coli, $C$. freundii FA 29, and $K$. pneumoniae P89 became capable of elaborating an ELISA-positive ST. On the contrary, $K$. pneumoniae AL55 showed positive results in the SMA, but not in the ELISA test. Transfer frequency ranged from approximately $1 \times$ $10^{-2}$ to $1 \times 10^{-1}$.

To see whether the acquired ability to produce ST was itself transmissible to other bacterial species, the new ST-positive strains E. coli, $K$. pneumoniae P89, and $K$. pneumoniae AL55 were used as donors in the remating experiments. Results are reported in Table 2 . All matings gave origin to enterotoxigenic strains. Interestingly, the recipient incubated with $K$. pneumoniae AL55, which produced an ELISA-negative ST, became capable of producing an ELISA-positive ST.

Transformation experiments. Competent cells of $E$. coli $\mathrm{K} 12$,
Table 1. Results of mating experiments

\begin{tabular}{lllll}
\hline & \multicolumn{4}{c}{$\begin{array}{c}\text { Recipient } \\
\text { after mating }\end{array}$} \\
\cline { 3 - 4 } Donor (ST+) & \multicolumn{1}{c}{ Recipient (ST-) } & SMA ELISA & $\begin{array}{c}\text { Transfer } \\
\text { frequency }\end{array}$ \\
\hline C. freundii AG2ST & E. coli K12 Lac - rif & + & + & $13 \times 10^{-2}$ \\
C. freundii AG2ST & K. pneumoniae P89 & + & + & $1 \times 10^{-2}$ \\
C. freundii AG2ST & K. pneumoniae AL55 & + & - & $7 \times 10^{-2}$ \\
E. coli R18 & C. freundii FA29 & + & + & $8 \times 10^{-2}$ \\
E. coli R18 & K. pneumoniae P89 & + & + & $5 \times 10^{-2}$ \\
E. coli R18 & K. pneumoniae AL55 & + & - & $9 \times 10^{-2}$ \\
E. coli R19 & C. freundii FA29 & + & + & $1 \times 10^{-2}$ \\
E. coli R19 & K. pneumoniae P89 & + & + & $1.5 \times 10^{-2}$ \\
E. coli R19 & K. pneumoniae AL55 & + & - & $2 \times 10^{-2}$ \\
\hline
\end{tabular}

Table 2. Results of remating experiments

\begin{tabular}{lcccc}
\hline & \multicolumn{3}{c}{$\begin{array}{c}\text { Recipient after } \\
\text { mating }\end{array}$} & Transfer \\
\cline { 3 - 4 } \multicolumn{1}{c}{ Donor $(\mathrm{ST}+)$} & $(\mathrm{ST}-)$ & SMA & ELISA & frequency \\
\hline E. coli $\mathrm{K} 12$ & C.freundii & + & + & $9 \times 10^{-2}$ \\
K. pneumoniae P89 & C.freundii & + & + & $5 \times 10^{-2}$ \\
K. pneumoniae AL55 & C.freundii & + & + & $6 \times 10^{-2}$ \\
\hline
\end{tabular}

Table 3. Results of transformation experiments

\begin{tabular}{lcc}
\hline Transformed strain & SMA & ELISA \\
\hline K. pneumoniac AL55 & + & - \\
K. pneumoniae P89 & + & + \\
E. coli K12 & + & + \\
\hline
\end{tabular}

K. pneumoniae P89, and $K$. pneumoniae AL55 were transformed by inserting the ST-encoding plasmid pSLM004. Transformed $E$. coli and $K$. pneumoniae P89 cells were positive in both the SMA and the ELISA test, whereas $K$. pneumoniae AL55 showed positive results only in the SMA (Table 3).

Physicochemical characteristics. The physicochemical characteristics of enterotoxic activities of K. pneumoniae P89 and AL55, E. coli $\mathrm{R} 18, E$. coli $\mathrm{R} 19$, and $C$. freundii AG2ST are reported in Table 4. All toxins showed identical behavior under the conditions tested.

Time course. The time-course effect of ST produced by different enterobacteria is reported in Figure 1. Toxins produced by all strains, with the exception of $K$. pneumoniae AL55, gave positive results in the SMA over a period of $6 \mathrm{~h}$. $K$. pneumoniae AL55 ST showed a short-acting enterotoxic activity, with a sharp peak at $2.5 \mathrm{~h}$ postinoculation and a rapid normalization of the gut weight/body weight ratio thereafter.

Stability of acquired enterotoxic activity. ST production acquired by all recipients, with the exception of $K$. pneumoniac AL55, was stable over a range of 3 to $6 \mathrm{mo}$. Enterotoxic activity produced by $K$. pneumoniae AL55 was lost in $30 \mathrm{~d}$ by the majority of the strains tested, but some strains were found to be positive up to $1 \mathrm{y}$ after conjugation.

Serotyping of Klebsiella strains. K. pneumoniae P89 showed positivity for K54 antigen; $K$. pneumoniae AL55 was related to $\mathrm{K} 60, \mathrm{~K} 61$, and $\mathrm{K} 15$ antigens.

\section{DISCUSSION}

The interspecific transfer of virulence properties has been previously demonstrated using laboratory-constructed plasmids (18). We used wild strains, isolated from children with acute diarrhea of secretory type $(11,12)$, as donors in bacterial conjugal mating. Also, the recipients were clinical isolates.

All the strains used in the conjugation experiments were able to transfer their toxigenic ability. This suggests that a plasmid exchange network exists among different enterobacteria. The same results obtained with conjugal mating experiments were 
Table 4. Characteristics of enterobacterial ST

\begin{tabular}{|c|c|c|c|c|c|}
\hline & $\begin{array}{c}\text { E. coli } \mathrm{ST} \\
18 \text { amino } \\
\text { acid }\end{array}$ & $\begin{array}{c}\text { E. coli } \mathrm{ST} \\
19 \text { amino } \\
\text { acid }\end{array}$ & $\begin{array}{l}\text { C. freundii } \\
\text { AG2ST }\end{array}$ & $\begin{array}{c}\text { K. pneumoniae } \\
\text { AL55 }\end{array}$ & $\begin{array}{c}\text { K. pneumoniae } \\
\text { P189 } \\
\end{array}$ \\
\hline Molecular weight & 1972 & 2000 & 2000 & $2000-10000$ & $2000-10000$ \\
\hline $\mathrm{pH}$ inactivation & $>8$ & $>8$ & $>8$ & $>8$ & $>8$ \\
\hline Methanol solubility & + & + & + & + & + \\
\hline 2-Mercaptoethanol sensitivity & + & + & + & + & + \\
\hline
\end{tabular}

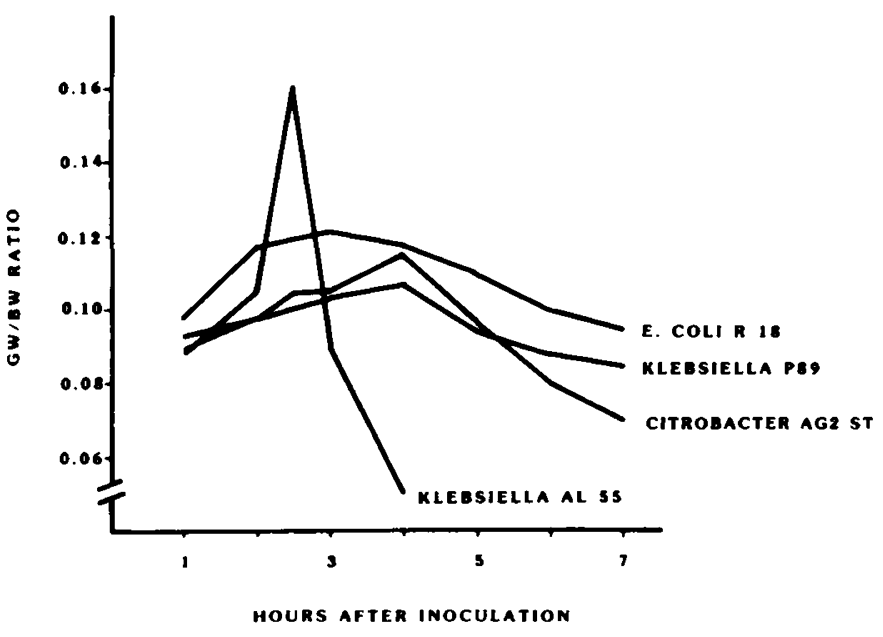

Fig. 1. Time course of enterotoxic activities of $E$. coli $\mathrm{R} 18$, Klehsiella P89, Klebsiella AL55, and Citrobacter AG2ST. Culture supernatant $(0.1$ $\mathrm{mL}$ ) was injected intragastrically into mice. The animals were killed at various times and the gut weight/body weight ratio $(\mathrm{GW} / \mathrm{BW})$ determined. A GW/BW ratio greater than 0.083 was considered positive. Each point represents the mean of three separate experiments.

also obtained when ST-negative bacterial cells were transformed by inserting a plasmid carrying the gene for ST. This provides reliable evidence for $\mathrm{ST}$ gene transfer among different enterobacteria rather than the transfer of some activity that regulates expression of ST gene.

The fact that all the strains used were able to transfer indicates the lack of specific restriction systems among wild strains with regard to toxigenic transfer. The transfer frequencies were in the same range for all the strains used, i.e. approximately $1 \times 10^{-2}$. Such frequency is in the upper range of that reported using strains transformed with laboratory-constructed plasmids (18). Recipients acquiring the ability to produce ST were also capable of transferring the toxigenic ability to other strains. This suggests that toxin conjugal transfer should be regarded as an infectious characteristic of enterobacteria.

It has been reported that toxins produced by other enterobacteria may be less stable than that produced by $E$. coli $(19)$. This appears not to be an absolute finding, based on our results. It should be noted, however, that stability of ST production may depend on storage conditions of individual bacterial species.

Like the other strains, $K$. pneumoniae AL55 acquired the ability to elaborate an enterotoxic activity by conjugation, but the phenotype of such ST was different from that produced by the donor, as it was not recognized by MAb. When K. pneumoniae AL55 was used as donor in the remating experiments, the recipient strain became capable of elaborating an ELISA-positive ST. This suggests that the modification might occur during the processing and/or exporting of the toxin, because it does not involve the transfer of genetically encoded activities capable of modifying the toxin expression from the donors.

The modification did not affect the main physicochemical characteristics of ST, which were identical for all the strains and compatible with that of ST1 or STa. However, the time-course effect of $K$. pneumoniae AL55 ST was markedly different from that of ST produced by all the other bacteria, showing a sharper peak of shorter duration. This observation has also been reported in the original work describing the toxin produced by $K$. pneumoniae AL55 (12). It is therefore clear that the modification of ST structure, probably occurring at the site recognized by the $\mathrm{MAb}$, results in variations of the kinetics of Klebsiella enterotoxic activity.

Only one of the two strains of Klebsiella used in the experiments showed the ability to modify the toxin, suggesting that this is a property of selected strains. As a consequence, different strains of $K$. pneumoniae can produce different types of ST.

In conclusion, transfer of toxigenic ability may occur in vitro between different enterobacteria. Selected Klebsiella strains may modify toxin expression.

These findings may have relevant implications in the field of basic knowledge related to ST production, but they are also of clinical interest. First, the transfer of toxigenic capability may explain the presence of multiple toxigenic strains in a child with diarrhea. Furthermore, the production of a modified ST toxin, as in the case of $K$. pneumoniae AL55, suggests that diagnostic kits based on the use of MAb may give false-negative results. Finally, the possibility exists that a specific immune response, evoked by vaccine against classic ST, may not be protective versus strains elaborating modified toxic activities.

Acknowledgments. The authors thank Dr. R. A. Giannella for critical manuscript review and Fritz and Ida Orskov for serotyping of Klebsiella strains.

\section{REFERENCES}

1. Black RE, Brown KH, Becker S, Abdul Alim ARM, Huq I 1982 Longitudinal studies of infectious diseases and physical growth of children in rural Bangladesh. II. Incidence of diarrhea and association with known pathogens. Am J Epidemiol 115:315-324

2. Guarino A. Alessio M. Tarallo L, Fontana M, Iacono G, Gobio Casali L, Guandalini S 1989 Heat-stable enterotoxin produced by Escherichia coli in acute diarthoea. Arch Dis Child 64:808-813

3. Betley MJ, Miller VL, Mekelanos JJ 1986 Genetics of bacterial enterotoxins Annu Rev Microbiol 40:257-263

4. Thompson MR 1987 Escherichia coli heat-stable enterotoxins and their receptors. Pathol Immunopathol Res 6:279-286

5. Rasheed JK, Guzman-Verduzco LM, Kupersztoch YM 1990 Two precursors of the heat-stable enterotoxin of Escherichia coli: evidence of extracellular processing. Mol Microbiol 4:265-273

6. Cohen MB, Guarino A, Shukla R. Giannella RA 1989 Age-related differences in receptors for Escherichia coli heat-stable enterotoxin in the small and large intestine of children. Gastroenterology 94:367-373

7. Guarino A, Cohen M, Thompson M, Dharmasathaphorn K, Giannella RA 1987 T84 cell receptor binding and guanylate cyclase activation by Escherichia coli heat-stable enterotoxin. Am J Physiol 16:G775-G780

8. Takao T, Shimonishi Y, Kobayashi M. Nishimura O. Arita M, Takeda T, Honda T, Miwatani T 1985 Amino acid sequence of heat-stable enterotoxin produced by Vibrio cholerae non-01. FEBS Lett 193:250-254

9. Takao T, Tominaga N, Shimonishi Y 1984 Primary structure of heat-stable enterotoxin produced by Yersinia enterocolitica. Biochem Biophys Res Commun 125:845-851

10. Aimoto $\mathrm{S}$, Watanabe $\mathrm{H}$. Ikemura $\mathrm{H}$. Shimonishi $\mathrm{Y}$. Takeda $\mathrm{T}$. Takeda $\mathrm{Y}$. Miwatani T 1983 Chemical synthesis of a highly potent and heat-stable analog of an enterotoxin produced by a human strain of enterotoxigenic Escherichia coli. Biochem Biophys Res Commun 112:320-326

11. Guarino A, Capano G, Malamisura B. Alessio M, Guandalini S, Rubino A 1987 Production of Escherichia coli STa-like heat-stable enterotoxin by Citrohacter freundii isolated from humans. J Clin Microbiol 25:110-114

12. Guarino A, Guandalini S. Alessio M. Gentile F, Tarallo L. Capano G. Migliavacca M, Rubino A 1989 Characteristics and mechanism of action of a heat- 
stable enterotoxin produced by Klebsiclla pne'umoniac from infants with secretory diarrhea. Pediatr Res 25:514-518

13. Guarino A. Giannella R. Thompson M 1989 (itrohacter frelundii produces an 18-amino acid heat-stable enterotoxin identical to the 18-amino acid Eschcrichia coli heat-stable enterotoxin (ST Ia). Infect Immun 57:649-652

14. Moseley SL. Hardy JW. Huq MI. Echeverria P. Falkow S 1983 Isolation and nucleotide sequence determination of a gene encoding a heat-stable enterotoxin of Escherichia coli. Infect Immun 39:1167-1174

15. Giannella RA 1976 Suckling mouse model for detection of heat-stable $E$. coll enterotoxin: characteristics of the model. Infect Immun 14:95-99

16. Thompson MR. Brandwein H, Labine-Recke M. Giannella RA 1984 Simple and reliable enzyme-linked immunosorbent assay with monoclonal antibodies for detection of Escherichia coli enterotoxin. J Clin Microbiol 20:59-64

17. Cohen SN, Chang ACY, Hsu L 1972 Nonchromosomal antibiotic resistance in bacteria: genetic transformation of $E$. coli by R-factor DNA. Proc Natl Acad Sci USA 69:2110-2114

18. Yamamoto T, Honda T, Miwatani T, Yokota T 1984 A virulence plasmid in Escherichia coli enterotoxigenic for humans: intergenetic transfer and expression. J Infect Dis 1 50:688-699

19. Back E, Molby R, Kaijser B, Stintzing G, Wadstrom T, Habte D 1980 Enterotoxigenic E. col $i$ and other gram-negative bacteria of infantile diarrhea: surface antigens, hemagglutinins, and loss of enterotoxigenicity. J Infect Dis $142: 318-327$

\section{Announcement}

The National Council of Jewish Women Center for the Child, in collaboration with the Society for Research in Child Development, has been awarded the contract by The Administration on Children, Youth and Families to hold the second national Head Start research conference, entitled "Translating Research into Practice: Implications for Serving Families with Young Children," on November 4-7, 1993 in Washington, DC. Following the success of the first Head Start research conference, "New Directions in Child and Family Research: Shaping Head Start in the Nineties," the major goals of the second conference are to identify and disseminate current research in early childhood and families, discuss child and family issues, and identify new research and program needs. A special focus will be on translating research into practice in an effort to help practitioners serving lowincome families gain a better understanding of effective early childhood interventions and programming. For additional information, contact Faith Lamb Parker, Ph.D., Project Director, Head Start Research Projects, NCJW Center for the Child, 53 West 23rd Street, New York, NY 10010, (212) 645-4048. 